\title{
Optimization of Location Allocation of Web Services Using A Modified Non-dominated Sorting Genetic Algorithm
}

\author{
Boxiong Tan, Hui Ma, Mengjie Zhang \\ School of Engineering and Computer Science, \\ Victoria University of Wellington, New Zealand \\ \{Boxiong.Tan, Hui.Ma, Mengjie.Zhang\}@ecs.vuw.ac.nz
}

\begin{abstract}
In recent years, web services technology is becoming increasingly popular because of the convenience, low cost and capacity to be composed into high-level business processes. The service location-allocation problem for a web service provider is critical and urgent, because some factors such as network latency can make serious effect on the quality of service (QoS). This paper presents a multi-objective optimization algorithm based on NSGA-II to solve the service location-allocation problem. A stimulated experiment is conducted using the WS-DREAM dataset. The results are compared with a single objective genetic algorithm (GA). It shows NSGA-II based algorithm can provide a set of best solutions that outperforms genetic algorithm.
\end{abstract}

\section{Introduction}

Web Services are considered as self-contained, self-describing, modular applications that can be published, located, and invoked across the Web [25]. In recent years, web services technology is becoming increasingly popular because of the convenience, low cost [1] and capacity to be composed into high-level business processes.

With the ever increasing number of functional similar web services being available on the Internet, the web service providers (WSPs) are trying to improve the quality of service (QoS) to become competitive in the market. QoS, also known as non-functional requirements to web services, is the degree to which a service meets specified requirements or web service users' needs [33, such as response time, security and availability. Among numerous QoS measurements, service response time is a critical factor for many real-time services, e.g. traffic service or finance service. Service response time has two components: transmission time (variable with message size) and network latency [18. Study [17] shows that network latency is a significant component of service response delay. Ignoring network latency will underestimate response time by more than 80 percent [26], since network latency is related to network topology as well as physical distance [14. To reduce the network latency WSPs need to allocate services location where has the lower latency to the user center that access the services. User center denotes a geometric location (e.g., a city) that is encompassed by a service 
area. Service area, also known as trade area, is a region where web service users concentrated at. There are many ways to identify service areas such as using IP information or user generated location data [23. Ideally, WSPs could deploy their services to each user center in order to provide the best quality. However, the more services deployed, the better the quality and the higher cost.

The Web service location-allocation problem is essentially a multi-objective optimization problem [3], for which there are two conflict objectives, to provide optimal QoS to web service users and to consume minimal deployment cost. This problem can be classified as a multidimensional knapsack problem (MKP), therefore, it is considered NP-hard due to the fact that the combinatorial explosion of the search space 27 .

Very few researches have studied the service location-allocation problem and most of the researchers treat this problem as a single objective problem. [1] 26] try to solve the problem by using integer linear programming techniques. In particular, 26] solved this problem by employing greedy and linear relaxations of Integer transpotation problem. However, integer programming (IP) is very effective for small-scale or mid-scale MKP but suffers from large memory requirement for large-scale MKP [15. Huang 12 proposed an enhanced genetic algorithm (GA)-based approach, which make use of the integer scalarization technique to solve this problem. This algorithm solves the problem with one objective and one constraint. However there are some deficiencies in the integer scalarization techniques [3. Firstly, decision makers need to choose appropriate weights for the objectives to retrieve a satisfactorily solution. Secondly, non-convex parts of the Pareto set cannot be reached by minimizing convex combinations of the object functions.

So far, to the best of our knowledge, there is no research has considered the service location-allocation problem as a multi-objective problem. Therefore, this paper we will treat service location-allocation problem as a multi-objective problem. Evolutionary multi-objective optimization (EMO) methodologies is ideal for solving multi-objective optimization problems [7, since EMO works with a population of solutions and a simple EMO can be extended to maintain a diverse set of solutions. With an emphasis for moving toward the true Pareto-optimal region, an EMO can be used to find multiple Pareto-optimal solutions in one single simulation run [19. Among numerous EMO algorithms, Non-dominated sorting GA (NSGA-II) [4, Strength Pareto Evolutionary Algorithm 2 (SPEA-2) [5] have become standard approaches. Some schemes based on particle swarm optimization (PSO) approaches are also proposed [10] [13]. NSGA-II is one of the most widely used methods for generating the Pareto frontier, because it can keep diversity without specifying any additional parameters [6]. In this paper, we propose to use NSGA-II to solve the web service location-allocation problem, which has two objectives, to minimize cost and deployment network latency.

The aim of this project is to propose a NSGA-II based approach to produce a set of near optimal solutions of service location-allocation, so that cost and overall network latency are close to minimum. Then, the WSPs could use the 
algorithm which proposed by this paper, to select an optimal plan based on their funds. The main objectives are:

- To model the web service location-allocation problem so that it can be tackled by NSGA-II.

- To develop a NSGA-II based approach to the web service location-allocation problem.

- To evaluate our proposed approach using some existing datasets.

In Section 2 we introduce the background of NSGA-II and GA. In Section 3 we provide models of the service location allocation problems. Section 4 develops a NSGA-II based algorithm. The experimental design and results evaluation are shown in Section 6. Section 7 provides a brief summary.

\section{Background}

GA 21 is a powerful tool to solve combinatorial optimization problems. It is an iterative procedure based on a constant-size population. In a GA, a population of strings (called chromosomes or the genotype of the genome), which are encoded as candidate solutions (called individuals, creatures, or phenotypes) to an optimization problem, evolves towards better solutions. Each genome is associated with a fitness value based on a fitness function that indicates how close it comes to meeting the overall specification, when compared to other genomes in the population. The fitness value of an individual is also an indication of its chances of survival and reproduction in the next generation. A typical genetic algorithm requires a genetic representation of the solution domain and a fitness function to evaluate the solution domain. Since a chromosome from the population represents a solution, when the algorithm starts, the whole population moves like one group towards an optimal area so the GA searches from a population of solutions rather than a single solution. Integer scalarization technique $[3$ is used to solve multi-objective problems with GA. It predefines a weight for each objective.

NSGA-II is a multi-objective algorithm based on GA. When used for problems with only two objectives, NSGA-II performs relatively well in both convergence and computing speed. However, NSGA-II has been criticized for its high computational cost and bad performance on applications with more than two objectives 8.

NSGA-II permits a remarkable level of flexibility with regard to performance assessment and design specification. NSGA-II assumes that every chromosome in the population has two attributes: a non-domination rank in the population and a local crowding distance in the population. The goal of NSGA-II is to converge to the Pareto front as possible and with even spread of the solutions on the front by controlling the two attributes.

The algorithm starts with a random initialization population. Once the population is sorted based on non-domination sorting, a rank is assigned to each chromosome. Then, a parameter called crowding distance is calculated for each individual. The crowding distance is a measure of how close an individual is to 
its neighbors. A large average crowding distance will result in better diversity in the population.

Parents are selected from the population by using tournament selection based on the rank and the crowding distance. An individual is selected in the rank if it is smaller than the other or if the crowding distance is greater than the other. The selected population generates offsprings using crossover and mutation operators.

The population with the current population and current offsprings is sorted again based on non-domination and only the best $\mathrm{N}$ individuals are selected, where $\mathrm{N}$ is the population size. The selection is based on rank and the on crowding distance on the last front.

\section{Problem Description and Modeling}

In this section, we first describe the service location-allocation problem, then we will present models for the services location allocation problem.

\subsection{Problem Description and Assumptions}

Web service location-allocation problem is to determine reasonable locations for web services so that the deployment cost of WSP can be minimized while service performance can be optimized. In this paper, to optimize service performance we consider to minimize network latency.

The task of service location allocation has two objectives:

- To minimize the total cost of the services.

- To minimize the total network latency of the services.

To model the service location-allocation problem, we consider the following assumptions:

Stakeholder Web Service Providers We consider the problem faced by a WSP who has existing facilities but wishes to use the collected data to re-allocate their services in order to maximum their profit.

The WSP must decide on facility locations from a finite set of possible locations. In order to make a decision, the WSP must first analyze the data collected from current use of services. The collected data should include the records of invocations from each unique IP address. Therefore, based on these data, the WSP could summarize several customer demands concentrated on $n$ discrete nodes [1, namely user centers. We assume that the WSP has already done this step and a list of user centers and candidate locations are given. Candidate location is the geometric location that is suitable to deploy services. Candidate locations are selected based on other criterions such as facilities or deployment cost. User centers and candidate locations can be overlapping, in fact, since web serivce users receive best QoS services if the web serivces are deployed locally. Therefore, the WSPs would like to choose user centers as candidate locations. In addition to deciding locations to of the services, information about network latency between user centers and candidate locations are needed.

The list below shows some critical information that should be providered by the WSPs.

1. A list of user centers 
2. A list of candidate locations

3. Service invocation frequency from user centers to services

4. Average network latency from user centers to candidate locations

5. Web service deployment cost for each candidate location

Worth nothing that service invocation frequency are changing over time. For example, a service was popular in some regions may be unfrequented after a few months. That's the main reason for WSPs re-allocate their services. Network latency highly depends on the network traffic and may be very different during periods of a day. However, as long as there is no significant changes in the network topology, the average network latency remain stable. Therefore, the average network latency for a period of time should be representative.

These are the main input data that the decision making is dependent on. The details of these input data and modeling are introduced in Section 3.2 Section 6 discussed the data that we used in the experiment.

Static deployment vs. Dynamic deployment As virtual machine technology and infrastracture-as-a-service (IaaS) are becoming more and more popular. Dynamic web service deployment become possible 20]. On the other hand, static deployment is still the mainstream because of a majority of web serivce are deployed on local infrastracture [11. In this paper, we made an assumption that WSPs periodicaly change the web service deployment since the user centers are changing over time.

\subsection{Model Formulation}

To model service location-allocation problem, we need to make use of a set of matrices, to present input information and output solutions.

For service location-allocation problem, we need information of service usage, network latency, and service deployment cost to decide service location-allocation so that the overall network latency can be minimized with minimal deployment cost and within constraints. Assume a set of $S=\left\{s_{1}, s_{2}, \ldots s_{s}, s_{x}\right\}$ services are requested from a set of locations $I=\left\{i_{1}, i_{2}, \ldots i_{i}, i_{y}\right\}$. The service providers allocate services to a set of candidate facility locations $J=\left\{j_{1}, j_{2}, \ldots j_{j}, j_{z}\right\}$.

In this paper, we will use the following matrices.

\begin{tabular}{ll}
\hline Matrices \\
$L$ & server network latency matrix $L=\left\{l_{i j}\right\}$ \\
$A$ & service location matrix $A=\left\{a_{s j}\right\}$ \\
$F$ & service invocation frequency matrix $F=\left\{f_{i s}\right\}$ \\
$C$ & cost matrix $C=\left\{c_{s j}\right\}$ \\
$R$ & user response time matrix $R=\left\{r_{i s}\right\}$ \\
\hline
\end{tabular}

service invocation frequency matrix, $F=\left[f_{i s}\right]$, is used to record services invocation frequencies from user centers, where $f_{i s}$ is an integer that indicates the number of invocations in a period of time from a user center to a service. For example, $f_{13}=85$ denotes service $s_{1}$ is called 85 times in a predefined period of time. 


$$
\begin{aligned}
& \begin{array}{ccccccc}
s_{1} & s_{2} & s_{3} & j_{1} & j_{2} & j_{3}
\end{array} \\
& F=\begin{array}{l}
i_{1} \\
i_{2} \\
i_{3}
\end{array}\left[\begin{array}{ccc}
120 & 35 & 56 \\
14 & 67 & 24 \\
85 & 25 & 74
\end{array}\right] \quad L=i_{2}\left[\begin{array}{ccc}
0 & 5.776 & 6.984 \\
5.776 & 0 & 2.035 \\
0.984 & 1.135 & 2.3
\end{array}\right]
\end{aligned}
$$

network latency matrix $L=\left[l_{i j}\right]$, is used to record network latencies from user centers to candidate locations. For example, the network latency between user center $i_{2}$ with candidate location $j_{1}$ is $5.776 \mathrm{~s}$. These data could be collected by monitoring network latencies 31 [32.

The cost matrix, $C=\left[c_{s j}\right]$, is used to record the cost of deployment of services to candidate locations, where $c_{s j}$ is an integer that indicates the cost of deploying a service to a location. For example, $c_{12}=80$ denotes the cost of deploying service $s_{1}$ to location $j_{2}$ is 80 cost units.

$$
\begin{aligned}
& \begin{array}{lll}
j_{1} & j_{2} & j_{3}
\end{array}
\end{aligned}
$$

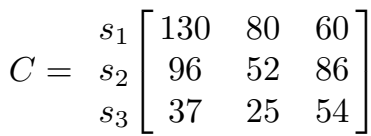

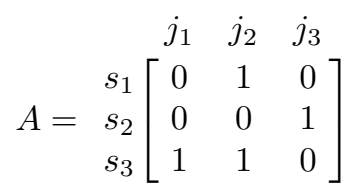

service location-allocation matrix $A=\left[a_{s j}\right]$ represents the actual service locationallocation, where $a_{s j}$ is a binary value 1 or 0 to indicate whether a service is allocate to a location or not.

Using service location allocation matrix $A=\left[a_{s j}\right]$ and network latency matrix $L=\left[l_{i j}\right]$, we can compute user response time matrix $R=\left[r_{i s}\right]$,

$$
r_{i s}=\operatorname{MIN}\left\{l_{i j} \mid j \in\{1,2, \ldots, z\} \text { and } a_{s j}=1\right\}
$$

For example, we can use the two example matrices $L$ and $A$ presented above to construct the response time matrix $R$. For each service $s$, by checking matrix $A$, we can find out which location the service has been deployed. Then we check matrix $L$, to find out its corresponding latency to each user center $i$. If there is more than one location, then the smallest latency is selected. Therefore, we can construct the response time matrix $R$ as:

$$
R=i_{1}\left[\begin{array}{ccc}
s_{1} & s_{2} & s_{3} \\
i_{2} & i_{3} \\
0 & 2.984 & 0 \\
1.135 & 2.035 & 0 \\
& & 0.984
\end{array}\right]
$$

\section{Summary}

\section{Input:}

- L: network latency from user centers to candidate locations

- F: service invocation frequency from user centers to web services

- $C$ : deployment cost for services in different candidate locations

\section{Temporary variable:}

- $R$ : user response matrix is a temporary variable generated during the optimization process 


\section{Output:}

- A: the solution matrix

\section{NSGA-II for Web Services Location Allocation}

To apply NSGA-II to the service location-allocation problem, the first step is to define the variable in NSGA-II, i.e., to identify chromosome, genetic operators and the fitness functions.

\subsection{Chromosome Representation and Constraints}

In our approach, we model the service location matrix $A=\left[a_{s j}\right]$ as a chromosome that we mentioned in Section 3 . The constraint setting is based on service providers' needs. One can set multiply constraints to the problem to narrow the potential searching space. In our case, we set two basic constraints. The first constraint service number constraint requires that each service is deployed in at least one location.

$$
\sum_{x \in S} a_{x j} \geq 1
$$

The second constraint, is cost constraint, which sets up the upper boundary of the total cost. An integer number CostLimitation is decided by the WSP.

$$
\sum_{s \in S} \sum_{j \in J} c_{s j} \times a_{s j} \leq \text { CostLimitation }
$$

\subsection{Genetic Operators}

The original NSGA-II uses a simulated binary crossover (SBX) 2] and polynomial mutation [24] to cope with continuous problems. However, our problem is discretized, therefore we use the regular GA mutation and crossover operations.

The selection operator is the tournament selection 28, which allows the highest probability of being reproduced to next generation.

Mutation The mutation operator works as follows: initially choose one random location from the chromosome. Then, this location is replaced by its inverse value, e.g., change 1 to 0 or change 0 to 1 . In this study, a chromosome in a population will be selected based on mutation possibility $P_{m}$, which is a parameter predefined and fixed.

Crossover The crossover operator in this paper is the single point crossover. The crossover is controlled by crossover probability $P_{c}$, is a parameter predefined and fixed. The crossover point is created randomly within the length of the chromosome.

\section{Fitness Function}

In order to accomplish these two objectives, we design two fitness functions to evaluate how good each chromosome meets the objectives. We use CostFitness to calculate the overall cost of deploying services under an allocation plan 


$$
\text { CostFitness }=\sum_{s \in S} \sum_{j \in J} c_{s j} \times a_{s j}
$$

where $c_{s j}$ is the cost of deploying service $s$ at location $j, a_{s j}$ represents the deployment plan. The sum of the multiplication of $c_{s j}$ and $a_{s j}$ is the total deployment cost.

We assume the latency is symmetrical between user center and candidate location. e.g., $l_{i j}=l_{j i}$

$$
\text { LatencyFitness }=\sum_{i \in I} \sum_{s \in S} r_{i s} \times f_{i s}
$$

Normalise function To indicate the goodness of an allocation solution we normalise CostFitness and LatencyFitness according to the largest and minimum values of CostFitness and LatencyFitness. Normalised fitness values can also be used to compare results from different approaches. Since the maximum and minimum values for total cost and total latency are deterministic, we use exhaustive search to find out the Latencymax. Latencymin is zero for we assume each service could be deployed in each user center. Cost $_{\min }$ is the cost of allocating each of services at a location that leads to the minimal cost and Cost $_{\max }$ is the cost of allocating each service is allocated to all the locations.

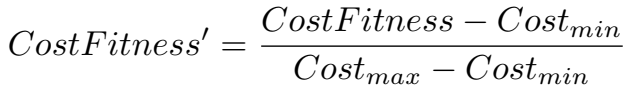

$$
\begin{aligned}
& \text { LatencyFitness }^{\prime}=\frac{\text { LatencyFitness }- \text { Latency }_{\min }}{\text { Latency }_{\max }-\text { Latency }_{\min }}
\end{aligned}
$$

\subsection{NSGA-II based algorithm for service location-allocation}

In this section we present our NSGA-II based algorithm for service location-allocation as Algorithm 1, comparing with the original NSGA-II our proposed algorithm has two new features.

Firstly, in order to avoid repeatly evaluating the fitness of chromosomes, after the first generation is initialized, it storing the Pareto front in the memory. In each generation, when evaluate the chromosome, the chromosome is checked to see it exists in the memory pool. If so, then the calculation of fitness will be skipped. At the end of each iteration, the Pareto front pool is updated to the current Pareto front. The reason for setting a memory pool is that, after a number of iterations, the population start converging. Most of the population are redundant. Therefore, a memory pool could considerably reduce the repeated computation.

Secondly, it uses general mutation and crossover operation instead of polynomial mutation and simulated binary crossover. It is important to note that the mutation and crossover operators can produce solutions that might violate the constraints. Therefore, repair operators are needed to try to maintain feasible solutions. The proposed algorithm checks the cost and service number constraint to avoid possible infeasible solutions. 


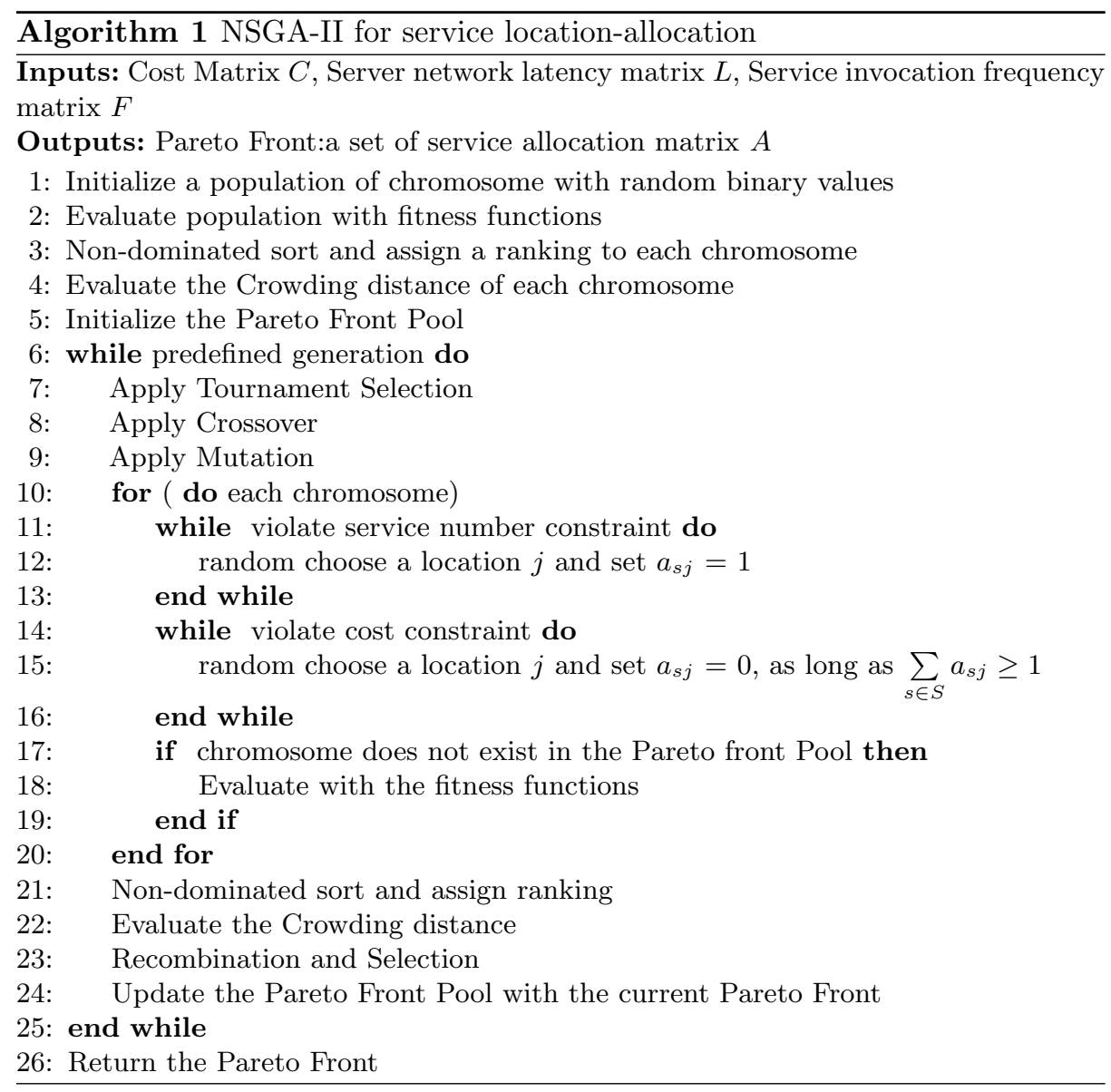

\section{GA for Web Service Location Allocation}

In order to show the advantages of our multi-objective NSGA-II based approach, we extend the single-objective GA based approach in [13] to consider two objectives. The extended GA-based approach is shown as Algorithm 2. We employ integer scalarization technique 9] to transform the multi-objective problem into a single objective problem. A weight $w$ needs to be predefined in GA. The weight measures the importance of objectives. Therefore, it is used to balance which objective is more favourable to the service provider.

Conventionally, the weight is in the range of $[0,1]$. For example, if we define the weight equals 0.7 . It denotes that we consider cost outweigh network latency. In our approach, we define the weight equals 0.5 since we consider both objectives equally important.

As in Section 3.2 we model an allocation matrix as a chromosome. Crossover and mutation operators are same as defined in Section 4.2. To evaluate the chromosomes of population. We use Integer Scalarization technique to 
calculate the fitness value.

$$
\text { Fitness }=w \times \text { CostFitness }^{\prime}+(1-w) \times \text { LatencyFitness }^{\prime}
$$

$w$ is a predefined value used to measure the important of cost and latency. Note that CostFitness and LatencyFitness are calculated using Formula 6 and 7 in section 4.2 .

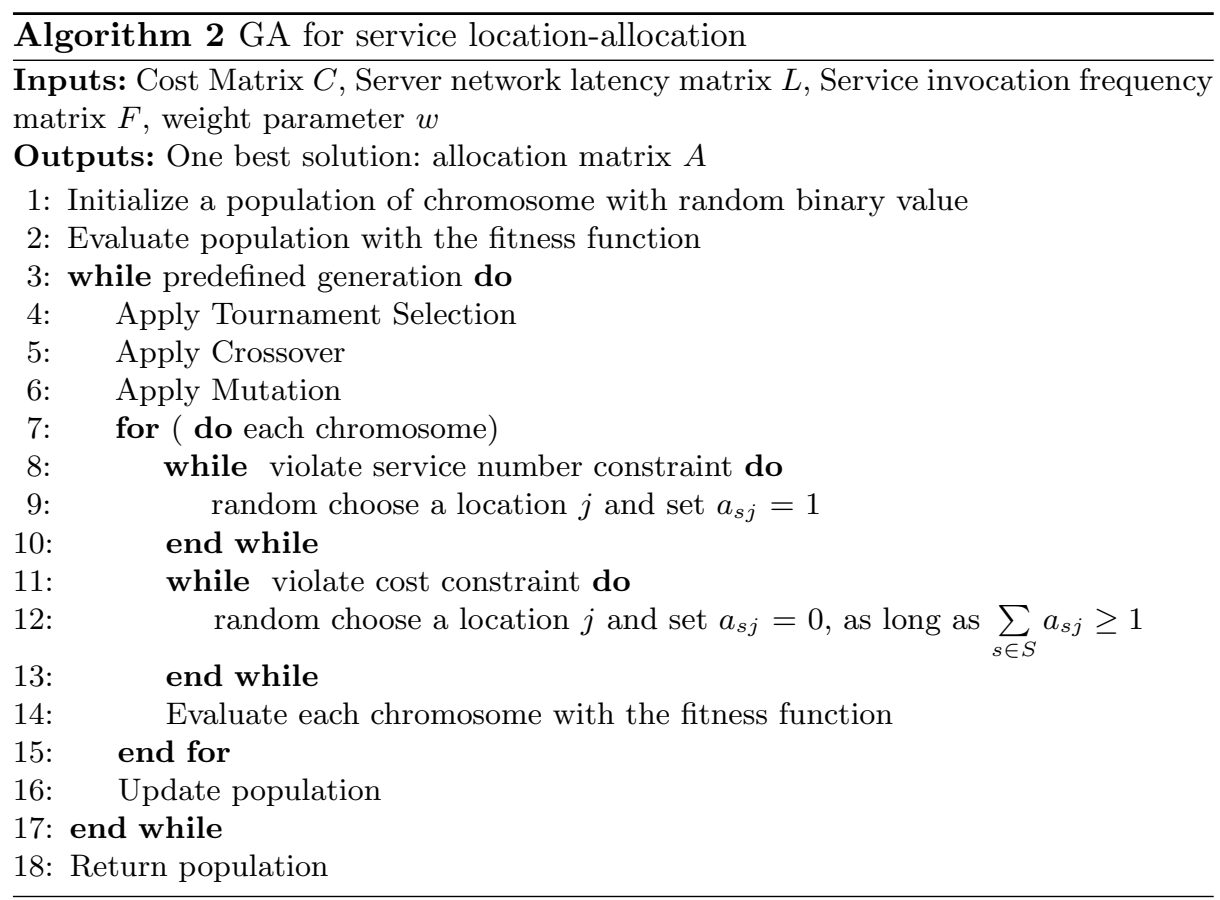

\section{Experiment Evaluation}

To evaluate the effectiveness and efficiency of our proposed NSGA-II based approach to service location-allocation. We compare our approach with the GA-based single objective approach in Section 5 using an existing dataset, WS-DREAM 31] 32, which is a historical dataset on QoS of Web services from different locations. It contains the data of latencies from 339 different user locations invoked 5824 web services scattered over different locations. The algorithm was coded in R 22] using existed package: NSGA2R. The program was run on a $3.40 \mathrm{GHz}$ desktop computer with 8 GB RAM. Four different service location-allocation problems are designed with different complexities. A cost matrix is randomly generated from a normal distribution with mean as 100 and standard deviation as 20. In addition, a frequency matrix, is randomly generated from a uniform distribution over $[1,120]$. 
Table 1: Test Cases

\begin{tabular}{|c|c|c|c|}
\hline problem & user location & server location & number of service \\
\hline 1 & 3 & 3 & 3 \\
\hline 2 & 5 & 5 & 5 \\
\hline 3 & 10 & 10 & 10 \\
\hline 4 & 15 & 15 & 15 \\
\hline
\end{tabular}

In each dataset, algorithms are run under four different levels of cost constraints: Sufficient condition (indicating 100\% the expected total cost), good condition $(70 \%)$, pool condition $(40 \%)$ and minimum budget condition $(0 \%)$. We try to stimulated a real world budget condition with these four scenarios. In the minimum budget condition, both algorithms exhaustively reduce the cost until it reaches the service number constraint. The NSGA-II based algorithm is runs 40 independent times with different random seeds ranging from 1 to 40. To test the efficiency of the algorithms, we evaluate the average run time for each algorithm.

Parameter settings for the algorithms are as follow. The population size is 50, and the maximum number of generations is 50 . The tournament size is 3 . The crossover probability $P_{c}$ is 0.8 and the mutation probability $P_{m}$ is 0.2 as we found that this combination can produce good results. We use same parameter settings for GA.

To compare the result of Algorithm 1 with GA-based algorithm (Algorithm 2), we first derive the Pareto front by using the approach in 29] [30], and then compare the results using approach in [16. In Xue's approach, NSGA-II generates 40 results (from 40 runs) under each cost constraint are presented in the Section 6.1. 40 sets of solutions achieved by each multi-objective algorithm are firstly combined into one set. GA also generates 40 sets of solutions, we select the best one based on its fitness value from each set and combine them into one set. The non-dominated solutions are presented to compare with the solutions achieved by GA.

In addition to the comparison between NSGA-II based algorithm and GA based algorithm, we conducted full experiments on NSGA-II with an initialisation and GA with an initialisation. We initialized the population with 49 random chromosomes and a chromosome which leading to minimum cost.

We expect the initialized algorithms superior than the uninitialized algorithms.

\subsection{Effectiveness comparison}

We conducted experiments on NSGA-II, GA, NSGA-II with initialisation and GA with initialisation respectively. We use cost fitness value and latency fitness value as $\mathrm{x}, \mathrm{y}$ coordinate. Our goal is to minimize both cost and latency.

Therefore, better solution should locate close to the origin.

Experimental results show that fewer different cost constraints leads to similar result patterns. Due to the page limitation we shows the results of one cost constraints. 


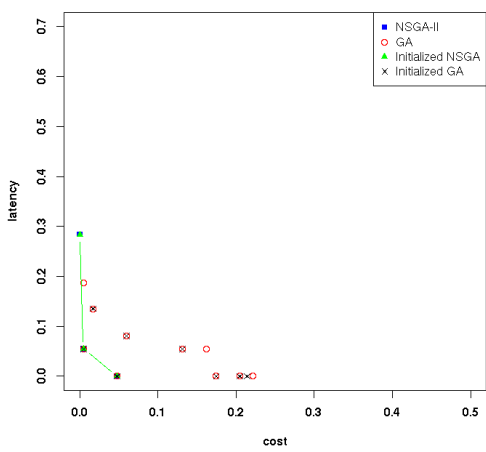

(a) $3 \times 3$

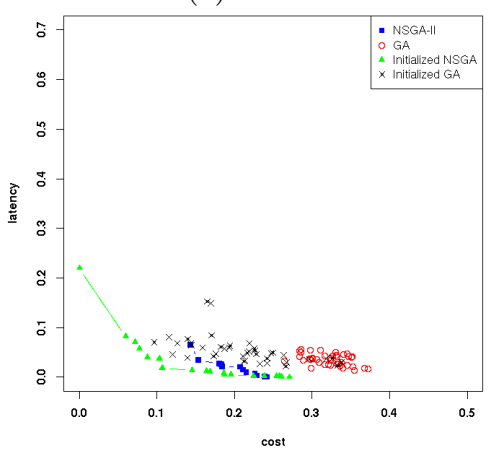

(c) $10 \times 10$

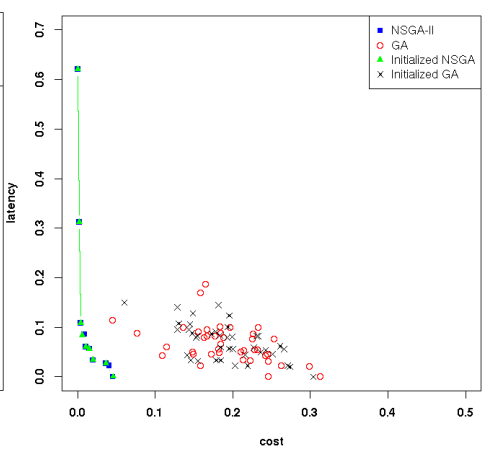

(b) $5 \times 5$

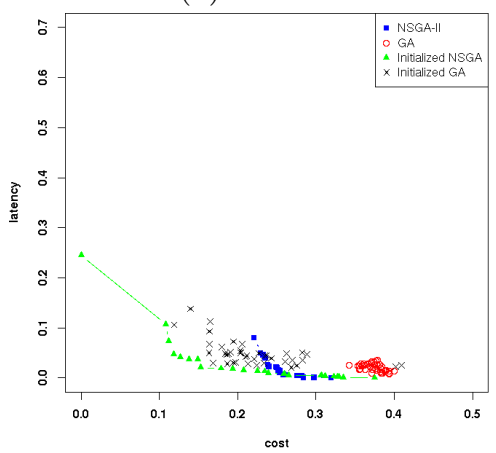

(d) $15 \times 15$

Fig. 1: Comparisons Between NSGA-II, GA, initialized-NSGA-II and initializedGA

From the above results we can see that for all the four problems, NSGA-II based approaches produce results that dominate or overlap the results from GA based approach. Further, NSGA-II with an initialized chromosome that represents service location-allocation of the minimum cost dominate the results without a chromosome of the lower cost, though for problem 1 and problem 2 of small complexity size, this observation is not obvious.

In particular, for big problems, problem 3 and 4, results of NSGA-II based approaches dominate the results of GA-based approaches. We also notice that even though the population size is small as 50, including a chromosome of optimal cost can help to narrow down searching space and to converge to optimal solution faster.

\subsection{Efficiency comparison}

The results from initialized algorithms are similar with uninitialized algorithms, therefore we only present the uninitialized results. As shown in the table above for small problems GA based approach are faster. However for bigger problem (problem 3 and 4) NSGA-II based approach, are more efficient than GA-based 
Table 2: Execution Time (s)

\begin{tabular}{|l|l|l|l|l|l|l|l|l|}
\hline & \multicolumn{2}{|l|}{ problem 1 } & \multicolumn{2}{l|}{ problem 2 } & \multicolumn{2}{l|}{ problem 3 } & \multicolumn{2}{l|}{4} \\
\hline & NSGA-II(s) & GA(s) & NSGA-II(s) & GA(s) & NSGA-II(s) & GA(s) & NSGA-II(s) & GA(s) \\
\hline Sufficient & $4.4 \pm 0.3$ & $1.6 \pm 0.1 \downarrow$ & $5.9 \pm 0.1$ & $3.2 \pm 0.1 \downarrow$ & $13.7 \pm 0.1$ & $11.0 \pm 0.1$ & $27.0 \pm 0.1$ & $23.9 \pm 0.5 \downarrow$ \\
\hline Good & $4.4 \pm 0.2$ & $1.6 \pm 0.1 \downarrow$ & $6.0 \pm 0.1$ & $3.2 \pm 0.1 \downarrow$ & $13.9 \pm 0.08$ & $11.1 \pm 0.3$ & $27.2 \pm 0.1$ & $24.1 \pm 0.27 \downarrow$ \\
\hline Poor & $4.6 \pm 0.19$ & $2.2 \pm 0.2 \downarrow$ & $6.3 \pm 0.07$ & $4.29 \pm 0.17 \downarrow$ & $15.2 \pm 0.16$ & $14.8 \pm 0.3$ & $31.3 \pm 0.28 \downarrow$ & $33.6 \pm 0.45$ \\
\hline Minimum & $4.6 \pm 0.1$ & $2.2 \pm 0.1 \downarrow$ & $7.2 \pm 0.12$ & $5.75 \pm 0.17 \downarrow$ & $24.12 \pm 0.5 \downarrow$ & $25.8 \pm 0.5$ & $56.72 \pm 1.6 \downarrow$ & $66.8 \pm 1.2$ \\
\hline
\end{tabular}

approach. Also NSGA-II based approach produces a set of non-dominated solutions instead of one solution, which provide WSPs with more options.

\section{Conclusion}

In this paper, we proposed a NSGA-II based approach to web service location-allocation problem. Our approach consider two objectives, minimizing cost and minimizing network latency at the same time. We have conducted a full experimental evaluation using the public WS-DREAM dataset to compare our approach to single-objective GA-based approach. The experimental results shows the NSGA-II based approach is effective to produce a set near-optima solutions for the web service location-allocation problem. Also, NSGA-II based approach are more efficient than GA-based approach for problem with big number of user centers and server locations. Future work will investigate the scalability of our proposed approaches for big datasets.

\section{References}

1. Aboolian, R., Sun, Y., Koehler, G.J.: A locationallocation problem for a web services provider in a competitive market. European Journal of Operational Research 194(1), $64-77$ (2009)

2. Beyer, H.G., Deb, K.: On self-adaptive features in real-parameter evolutionary algorithms. Evolutionary Computation, IEEE Transactions on 5(3), 250-270 (2001)

3. Caramia, M.: Multi-objective optimization. In: Multi-objective Management in Freight Logistics, pp. 11-36. Springer London (2008)

4. Deb, K., Pratap, A., Agarwal, S., Meyarivan, T.: A fast and elitist multiobjective genetic algorithm: NSGA-II. Evolutionary Computation, IEEE Transactions on 6(2), 182-197 (2002)

5. Deb, K., Mohan, M., Mishra, S.: Evaluating the epsilon-domination based multi-objective evolutionary algorithm for a quick computation of pareto-optimal solutions. Evol. Comput. 13(4), 501-525 (2005)

6. Deb, K., Sundar, J., N, U.B.R., Chaudhuri, S.: Reference point based multi-objective optimization using evolutionary algorithms. In: International Journal of Computational Intelligence Research. pp. 635-642. Springer-Verlag (2006)

7. Desai, S., Bahadure, S., Kazi, F., Singh, N.: Article: Multi-objective constrained optimization using discrete mechanics and nsga-ii approach. International Journal of Computer Applications 57(20), 14-20 (2012), full text available

8. Domnguez, J., Montiel-Ross, O., Seplveda, R.: High-performance architecture for the modified nsga-ii. In: Melin, P., Castillo, O. (eds.) Soft Computing 
Applications in Optimization, Control, and Recognition, Studies in Fuzziness and Soft Computing, vol. 294, pp. 321-341. Springer Berlin Heidelberg (2013), http://dx.doi.org/10.1007/978-3-642-35323-9_13

9. Ehrgott, M.: A discussion of scalarization techniques for multiple objective integer programming. Annals of Operations Research 147(1), 343-360 (2006), http://dx.doi.org/10.1007/s10479-006-0074-z

10. Elhossini, A., Areibi, S., Dony, R.: Strength pareto particle swarm optimization and hybrid EA-PSO for multi-objective optimization. Evol. Comput. 18(1), 127-156 (2010)

11. He, K., Fisher, A., Wang, L., Gember, A., Akella, A., Ristenpart, T.: Next stop, the cloud: Understanding modern web service deployment in ec2 and azure. In: Proceedings of the 2013 Conference on Internet Measurement Conference. pp. 177-190. IMC '13, ACM, New York, NY, USA (2013), http://doi.acm.org/10.1145/2504730.2504740

12. Huang, H., Ma, H., Zhang, M.: An enhanced genetic algorithm for web service location-allocation. In: Decker, H., Lhotsk, L., Link, S., Spies, M., Wagner, R. (eds.) Database and Expert Systems Applications, Lecture Notes in Computer Science, vol. 8645, pp. 223-230. Springer International Publishing (2014)

13. Huang, V.L., Suganthan, P.N., Liang, J.J.: Comprehensive learning particle swarm optimizer for solving multiobjective optimization problems: Research articles. Int. J. Intell. Syst. 21(2), 209-226 (2006)

14. Huffaker, B., Fomenkov, M., Plummer, D., Moore, D., claffy, k.: Distance Metrics in the Internet. In: IEEE International Telecommunications Symposium (ITS). pp. 200-202. IEEE, Brazil (Sep 2002)

15. Hwang, J., Park, S., Kong, I.Y.: An integer programming-based local search for large-scale maximal covering problems. International Journal on Computer Science and Engineering pp. 837-843 (2011)

16. Ishibuchi, H., Nojima, Y., Doi, T.: Comparison between single-objective and multi-objective genetic algorithms: Performance comparison and performance measures. In: Evolutionary Computation, 2006. CEC 2006. IEEE Congress on. pp. $1143-1150$ (2006)

17. Jamin, S., Jin, C., Kurc, A., Raz, D., Shavitt, Y.: Constrained mirror placement on the internet. In: INFOCOM 2001. Twentieth Annual Joint Conference of the IEEE Computer and Communications Societies. Proceedings. IEEE. vol. 1, pp. 31-40 vol.1 (2001)

18. Johansson, J.M.: On the impact of network latency on distributed systems design. Inf. Technol. and Management 1(3), 183-194 (2000)

19. Kanagarajan, D., Karthikeyan, R., Palanikumar, K., Davim, J.: Optimization of electrical discharge machining characteristics of wc/co composites using non-dominated sorting genetic algorithm (nsga-ii). The International Journal of Advanced Manufacturing Technology 36(11-12), 1124-1132 (2008)

20. Kemps-Snijders, M., Brouwer, M., Kunst, J.P., Visser, T.: Dynamic web service deployment in a cloud environment (2012)

21. Man, K.F., Tang, K.S., Kwong, S.: Genetic algorithms: concepts and applications. IEEE Transactions on Industrial Electronics 43(5), 519-534 (1996)

22. Morandat, F., Hill, B., Osvald, L., Vitek, J.: Evaluating the design of the R Language: Objects and functions for data analysis. In: Proceedings of the 26th European Conference on Object-Oriented Programming. pp. 104-131.

ECOOP'12, Springer-Verlag (2012) 
23. Qu, Y., Zhang, J.: Trade area analysis using user generated mobile location data. In: Proceedings of the $22 \mathrm{Nd}$ International Conference on World Wide Web. pp. 1053-1064. WWW '13, International World Wide Web Conferences Steering Committee, Republic and Canton of Geneva, Switzerland (2013), http://dl.acm.org/citation. cfm?id=2488388.2488480

24. Raghuwanshi, M.M., Kakde, O.G.: Survey on multiobjective evolutionary and real coded genetic algorithms. In: Proceedings of the 8th Asia Pacific symposium on intelligent and evolutionary systems. pp. 150-161 (2004)

25. Ran, S.: A model for web services discovery with QoS. SIGecom Exch. 4(1), 1-10 (2003)

26. Sun, Y., Koehler, G.J.: A location model for a web service intermediary. Decis. Support Syst. 42(1), 221-236 (2006)

27. Vanrompay, Y., Rigole, P., Berbers, Y.: Genetic algorithm-based optimization of service composition and deployment. In: Proceedings of the 3rd International Workshop on Services Integration in Pervasive Environments. pp. 13-18. SIPE '08, ACM (2008)

28. Xie, H., Zhang, M., Andreae, P., Johnson, M.: An analysis of multi-sampled issue and no-replacement tournament selection. In: Proceedings of the 10th Annual Conference on Genetic and Evolutionary Computation. pp. 1323-1330. GECCO '08, ACM (2008)

29. Xue, B., Zhang, M., Browne, W.N.: Multi-objective particle swarm optimisation (pso) for feature selection. In: Proceedings of the 14th Annual Conference on Genetic and Evolutionary Computation. pp. 81-88. GECCO '12, ACM (2012)

30. Xue, B., Zhang, M., Browne, W.: Particle swarm optimization for feature selection in classification: A multi-objective approach. Cybernetics, IEEE Transactions on 43(6), 1656-1671 (Dec 2013)

31. Zhang, Y., Zheng, Z., Lyu, M.: Exploring latent features for memory-based QoS prediction in cloud computing. In: Reliable Distributed Systems (SRDS), 2011 30th IEEE Symposium on. pp. 1-10 (2011)

32. Zheng, Z., Zhang, Y., Lyu, M.: Distributed QoS evaluation for real-world web services. In: Web Services (ICWS), 2010 IEEE International Conference on. pp. 83-90 (2010)

33. Zhou, J., Niemela, E.: Toward semantic QoS aware web services: Issues, related studies and experience. In: Web Intelligence, 2006. WI 2006. IEEE/WIC/ACM International Conference on. pp. 553-557 (2006) 